\title{
Types and Roles of Models in Adaptive Architecture
}

\author{
Marie Ulber ${ }^{1} \quad$ Mona Mahall $^{1} \quad$ Asli Serbest $^{2}$ \\ 1.Architecture and Art, HafenCity University Hamburg, Überseeallee 16, 20457 Hamburg, Germany \\ 2.Temporary Spaces, University of the Arts Bremen, Am Speicher XI 8, 28217 Bremen, Germany
}

\begin{abstract}
If adaptive architecture is both, a techno-spatial environment and a set of environmental behaviors and practices, its design requires the collaboration of diverse disciplines. During the process of collaboratively developing an adaptive high-rise, models turn out to be the central medium in the multidisciplinary teamwork. They contribute to disciplinary questions of development and realization; they also generate and transfer knowledge and act as translators by facilitating exchange and cooperation between disciplines. The present study examines tasks and relations from the first design model to different functional models for the adaptive high-rise. It shows the initial architectural model as an overall role model and vision. While functional models perform isolated dynamic tasks, analytical models are used for review and discussion.
\end{abstract}

Keywords: adaptive architecture, multidisciplinary research, design process, translation models, knowledge transfer

DOI: $10.7176 / \mathrm{ADS} / 82-04$

Publication date:May $31^{\text {st }} 2020$

\section{Introduction}

Adaptive architecture fundamentally changes our idea of buildings as well as our experience of and interaction with the built environment (Ulber, Mahall, Serbest, 2020). For architects, this type of architecture brings with it previously unknown opportunities and challenges. Adaptive buildings, which respond dynamically and in real time to changing environmental or user conditions, are still a recent development. So far, this includes adaptive structures, shells, façades, spatial configurations, furnishings or interior climate. Adaptive technology challenges the architectural design work and requires new approaches, methods and cooperation. Therefore this case study analyzes the roles and uses of diverse models in the Collaborative Research Center "Adaptive Skins and Structures for the Built Environment of Tomorrow" at the University of Stuttgart. It explores the relations of various model types of the demonstrator high-rise structure that is currently being built. The development of the adaptive highrise is the central project of the research group, requiring multidisciplinary cooperation and involving new interconnected design and development processes. In these processes, our initial hypothesis states that models play a crucial role as a medium between different disciplinary knowledge cultures: that is, between design and technological fields. The case study also provides insight into the design and development processes of an adaptive building and discusses the role of architecture and its models in a multidisciplinary project. Its results can therefore also point to a new design approach for adaptive architecture.

Buildings have always been adapted through users changing them according to their needs and current situation, e.g., tents that can be set up in different locations or with closable openings for ventilation or light. The term adaptivity was initially associated with the reuse and transformation of building structures into another relatively constant state, representing a linear adaptation sequence. This includes "adaptable architecture" which discusses various adaptable mechanisms and strategies, including adjustable, versatile, refitable, convertible, scalable and movable buildings (Schmidt III, Austin, 2016, p. 146). Active adaptation principles of buildings, however, include reaction of building structures, shells and interior design to current environmental and user requirements. Currently, the adaptation principles are designed and developed in advance, but the intensity of the adaptive action depends on the situation in real-time.

Active adaptive reactions vary. One option is to use materials with adaptive properties, such as in the HygroSkin: Meteorosensitive Pavilion by the Institute for Computational Design (ICD, 2013), in which thin wooden leaves open and close depending on humidity, thus providing an adaptive ventilation concept. The adaptive reactions can also be carried out by engines, as in the adaptive external façades of the Al Bahr towers. Sensors determine the wind and sun intensity and accordingly the façade elements are opened or closed by computer control (Hill, 2018, pp.118-121). Adaptive responses are linked to adaptation objectives, either environmental conditions and/or user needs. In the ECO-29 event room by FoxLin and Brahma Architects, 2012, the inner flexible shell of the room can be adapted with motors and allows a variety of spatial configurations depending on the event (Fox, 2016, pp. 106-13). In contrast, the Bengt Sjostrom Starlight Theatre by Studio Gang Architects in Rockfort Illinios, 2003, shows an adaptive reaction of the entire roof construction which opens the auditorium to the sky (Yiannoudes, 2016, pp.40-41). In the Sharifi-ha house in Tehran, Iran, designed by Nextoffice, 2013, an entire room per level can rotate out and thus shift the boundaries between inside and outside (Kolarevic, Parlac, 2015, p.7). The fact that traditionally rather immobile massive architectural elements become 
movable and flexible means a fundamental change. In this sense, the SmartShell built in 2012 by the Institute for Lightweight Construction at the University of Stuttgart represents a further developmental step. The four centimeter thick wooden shell with a span of $10 \mathrm{~m}$ has one fixed and three hydraulic base points to "compensate for both static and dynamic strains" (Sobek, 2016, p. 167). This makes former static elements dynamically flexible by reacting to different load cases depending on the situation. The buildings mentioned here mostly exhibit only one adaptation principle, but the discussed dimensions of adaptivity show that, in the future, buildings will be able to adapt to different environmental situations and uses. In addition to extending the lifetime of buildings, materials and energy are actively saved depending on the adaptation principles, thus conserving natural resources. In times of global ecological change, adaptive buildings enable a more sustainable and resilient architecture. But how does adaptive architecture change the way architects work? The model study on the design and development genesis of the adaptive demonstrator high-rise aims to provide new insights in this regard.

Models play an important role in the process of designing and planning architecture (Yaneva, 2009, 158). As representational media, models and drawings are essential tools in the architectural design process (Schilling, 2018, p. 31). Models are three-dimensional expressions of a design and are used initially for spatial exploration, examination and correction. Albena Yaneva (2005) analyzed in an ethnographic study at the Office for Metropolitan Architecture (OMA) the design process of the Whitney Museum addition with scaling models to find the best solution on various scales. The 'Prada Genealogy' shows the design genesis through models beginning with the urban positioning of a volume, interior and façade studies up to the final model of the Prada Store in Tokyo 2003 by the architects Herzog \& de Meuron (Ursprung, 2012). Both studies show architectural form-finding processes through models. The design genesis concludes with an architectural form that inseparably unites all the considerations and ideas of the designers with regard to building site, use, spatial structure, materials, etc. (Zumthor, 2006, 69). Finally, the finished design is presented in a model alongside drawings to convince the client or a competition jury. These "ideal" models are a communication medium to the outside world to illustrate the architectural idea. Today, architectural design work is done with both physical and digital models. For adaptive architecture, this design work with models is challenging because several states, appearances and forms must be designed. The question arises, how models in adaptive architecture projects take over what kind of tasks and uses?

\section{Adaptive high-rise as a common vision}

In the design, development and implementation of the adaptive demonstrator high-rise, a wide variety of disciplines are involved and working together. The investigated Collaborative Research Center's (CRC 2017) "Adaptive Skins and Structures for the built Environment of Tomorrow" at the University of Stuttgart pursues a sustainable and ecological architecture with regard to climate change and scarcity of raw resources. Based on lightweight construction, material and energy consumption are reduced and new adaptive concepts for an architecture of the future are developed. The demonstrator high-rise plays a central role in the research project, as all adaptive developments refer to it. With the adaptive supporting structure, the high-rise reacts actively to wind loads or earthquakes. Since actuators reduce maximum load cases, the construction can be reduced in dimension and material. Various adaptive components are developed, installed and tested in the high-rise including façade elements, shells and an adaptive indoor climate which react intelligently and adaptively to the environment and users. In addition, the control and reliability of adaptive concepts, as well as innovative methods for designing and implementing adaptive architecture must be developed.

The researchers involved in the demonstrator high-rise project and interviewed for the model study work in the disciplines of architecture, civil engineering, structural engineering, mechanical engineering, system dynamics, aerospace engineering, visualization technology, building physics and polymer chemistry. The project structure of the Collaborative Research Center is divided into four projects: (A) Design and planning methodology, (B) System engineering and design, (C) Integrative components and (D) Economic, ecological and socio-cultural aspects. With their sub-project "Architectural Design Concepts for Adaptive Skins and Structures", the authors belong to project A "Design and Planning" and regularly take part in project meetings and doctoral colloquia. The involved departments and researchers of the University of Stuttgart are spatially separated from each other on campus and in the city. In addition to direct collaborations for specific design solutions, the participants regularly exchange insights and progress at project meetings, doctoral colloquia and the yearly summer school. It is our hypothesis that models play a special role in this exchange, which is examined in this study. 

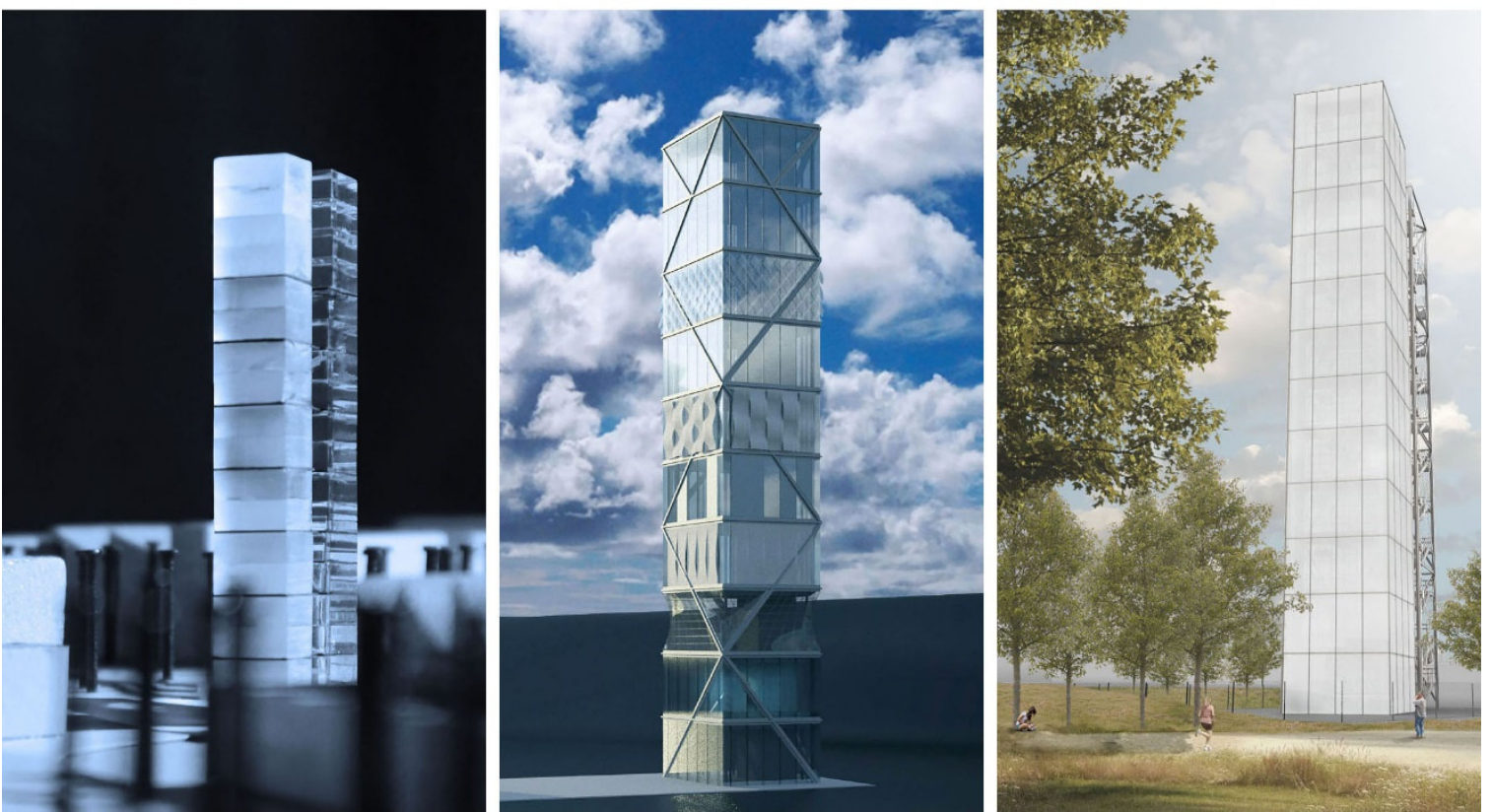

Figure 1. Adaptive high-rise building in model photo and 3D renderings. Credit: Institute for Lightweight Structures and Conceptual Design (ILEK), University of Stuttgart.

The design of the demonstrator high-rise envisions an adaptive structure that involves many disciplines in its development and realization. The first model photo shows the architectural design with the twinning of demonstrator and stair tower (Figure 1). The blue colored picture is atmospherically charged, the transparent acrylic glass tower shines. The visualization embodies the irreducible overall idea. In the second rendering some adaptive functions find their expression. The isolated demonstrator building with changing façades indicates that the experimental building will integrate various new adaptive developments. The third rendering shows the design of the first implementation of the demonstrator high-rise with a homogeneous façade. With the introduction to the research project including organisational structures, participating disciplines and the short presentation of the central building project, the background of this study is revealed. Starting from this point, the case study examines the use of various models in the development and implementation of the adaptive high-rise.
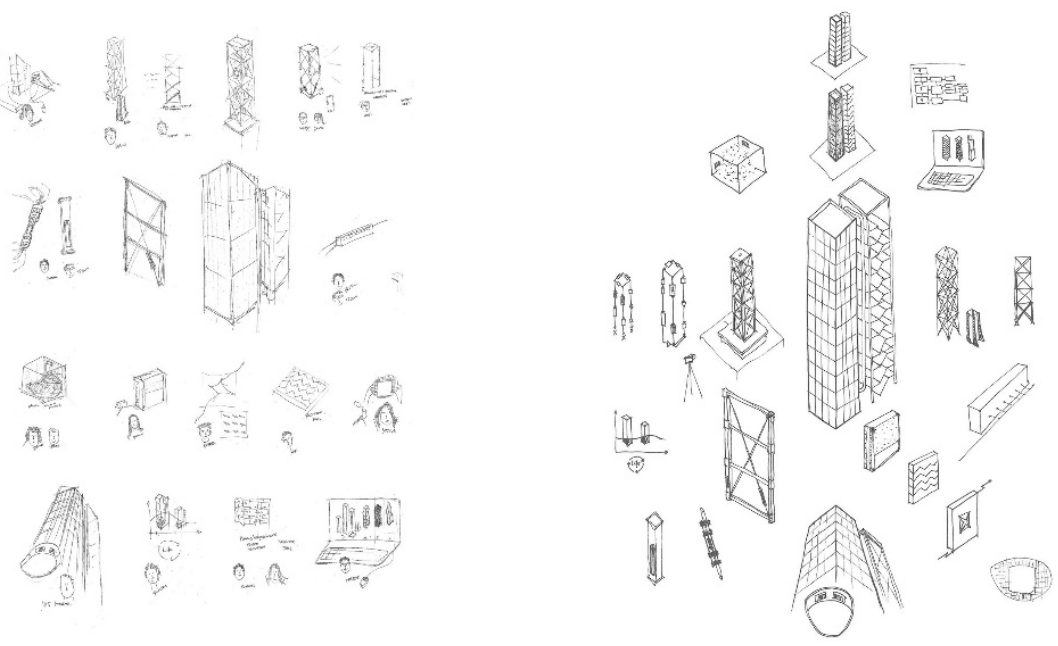

Figure 2. Translations of model findings into sketches and drawings.

\section{Methodological approach}

In the case study within the Collaborative Research Center, semi-structured interviews and interpretative graphic translation were combined as a methodological approach. The authors themselves are working on a sub-project on architectural concepts and regularly take part in project meetings and doctoral colloquia, which led to the hypothesis that the models are of particular importance in the multidisciplinary research project. In order to gain further insights, semi-structured interviews with colleagues were conducted (Helfferich, 2014, pp. 559-74). The 
researchers were asked about the use of models in their sub-project, including challenges or surprises, about the relationship to the demonstrator building, and about the type and role of their own and other models in exchange with other researchers. The interviews were conducted according to the principle "follow the actors" from the actor network theory, which is based on the assumption that human and non-human beings are equally involved in actions, thus also in design and research processes (Yaneva, 2009, pp. 23-27). With this approach, the question for contributions of the models to the design and development process of the demonstrator building were being kept open. Starting with interviews in our own project group, the selection was later based on information from respondents who often referred us directly to colleagues and models who were also involved in the design, development and implementation process of the demonstrator high-rise. A total of 20 out of 25 researchers were interviewed, which shows that the work of many participants is closely related to the demonstrator building. The results of the observations and interviews were translated into sketches. This form of interpretative research records the most important findings in graphics (Ulber, 2017, p. 84). Subsequently, the sketches were arranged in a first reference system showing the model types in the relational context (Figure 2). The processing of the sketches into drawings makes the research network found in this study readable for others. Every single drawing represents a model with certain characteristics that are revealed in the description. The results of this model study were elaborated both in discourse and in graphics. The study and the survey were communicated in project meetings, later first results and sketches of the model cosmos were shown and finally the drawings and results were presented. This enabled a discussion in which our results could be reviewed by our research colleagues (Döring, Bortz, 2016, p. 70).

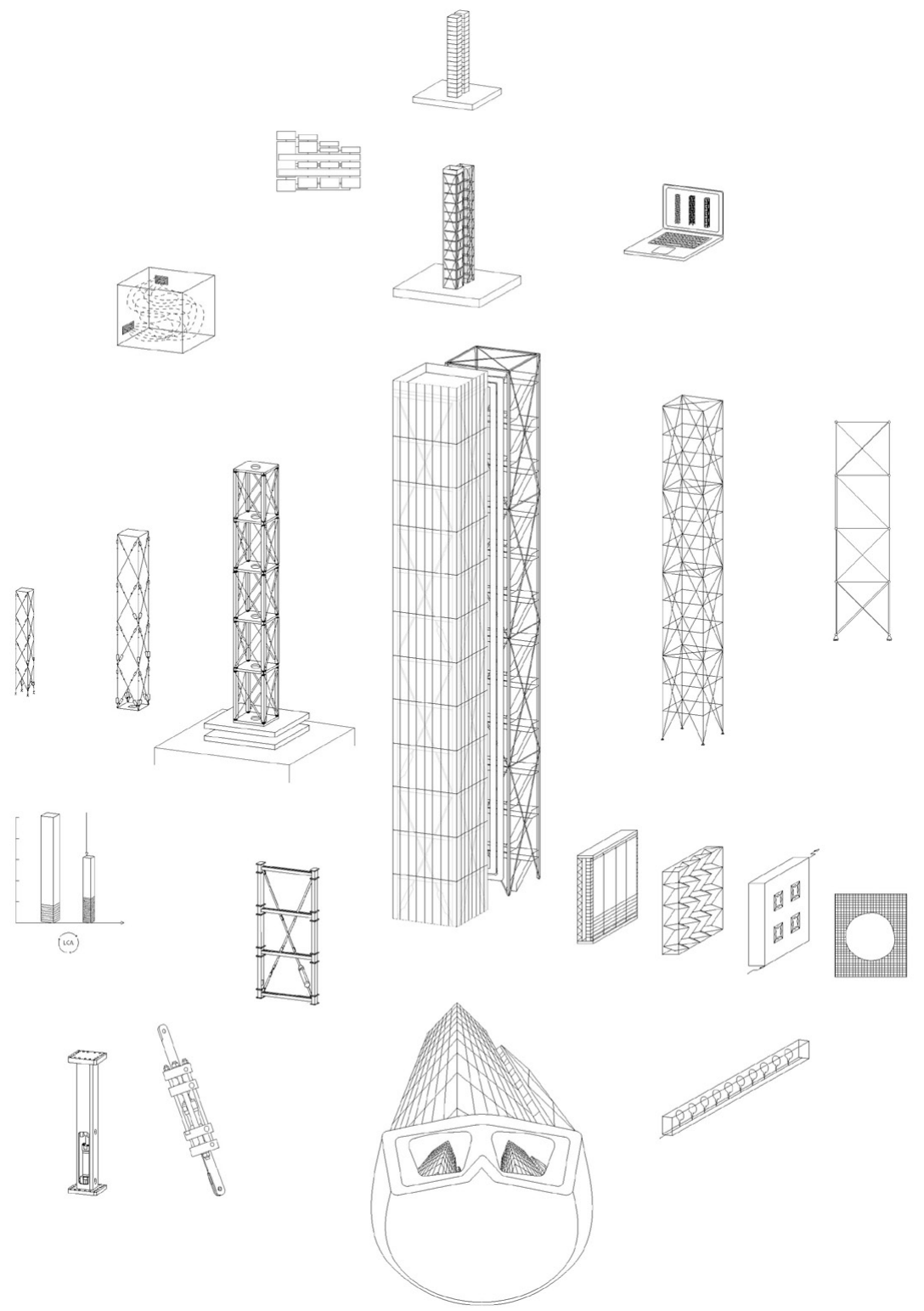

Figure 3. Model cosmos with analyzed models in relation to the demonstrator high-rise. 


\section{Model cosmos}

The model cosmos documents all models of the Collaborative Research Center found in the study in a relation to the demonstrator high-rise (Figure 3). The drawing gives an overview of the models developed without scaling and used in two and a half years of ongoing research. Like an abstract map, it shows detected references and connections of the models in their all-dimensional and simultaneous development. The vertical and horizontal axes display all models of the demonstrator building, the lower half gives space to models of developed adaptive components, and reflective and analytical models are presented as surrounding satellites. Understood as three categories, the individual models and their tasks as well as their relationship to the demonstrator high-rise are discussed separately. These include physical and digital design, calculation, simulation and functional models, mockups, samples and theoretical process models. Each individual model drawing unfolds its tasks, meaning and references in the description and discussion.

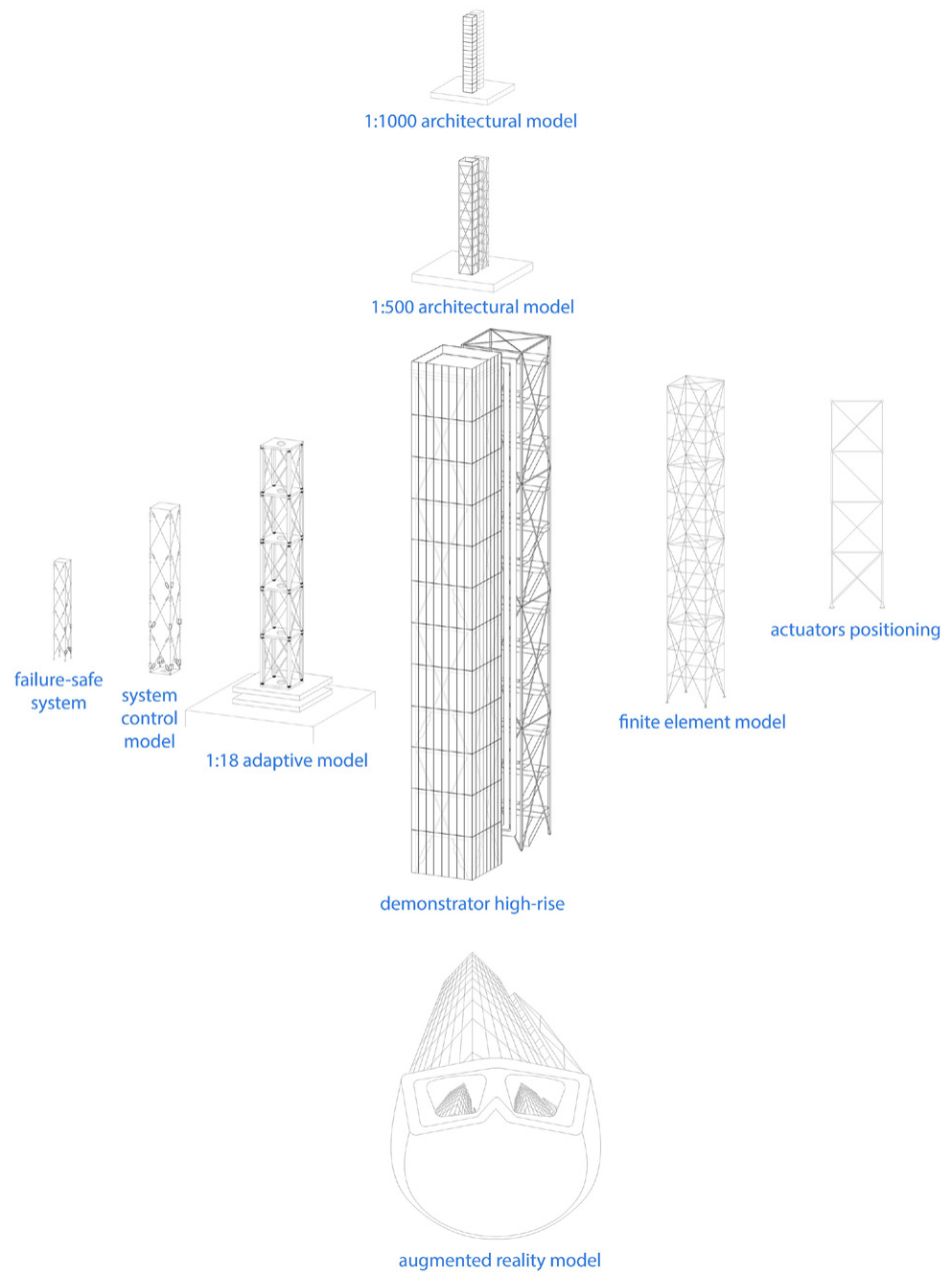

Figure 4. Models of the demonstrator high-rise in model cosmos.

4.1 Models of the demonstrator high-rise

The first consideration of the model cosmos focuses on complete models of the demonstrator building that were found in the model study (Figure 4). The architectural design models lead from the top down to the centrally depicted demonstrator high-rise. The first model, made of acrylic glass, is an urban planning model on a scale of 1:1000. This model and its blue photos serve as a vision and role model in the Collaborative Research Center (Figure 1, left). The architectural design presented in this model persists throughout the multidisciplinary planning process and is therefore understood as a positioning. This can be seen in the second architectural model made of wood in the scale of 1:500, which concretizes the design in terms of the number of floors and construction type. Both models stand in an urban context, which is not shown in the graphic, but can be seen for example on the model photo (Figure 1, left). Below the central demonstrator, the building model is viewed through augmented reality glasses that enable the wearer to experience the high-rise building in advance in a mixed reality, virtually in full size at the real construction site. The current rendering of the demonstrator building is based on the same 3D model (Figure 1, right). Both provide a preview of the appearance of the possible future. 
Horizontally, the drawing depicts the functional models found in this study of the demonstrator high-rise. The finite element model (FEM) shown to the right of the demonstrator building provides the necessary knowledge for the adaptive structure. The digital model of the load-bearing structure is used to calculate the occurring loads, bending and torsion, that means the deformation figure. The researcher also use this model to determine the position of the actuators, as shown on the right. To the left of the adaptive high-rise, the 1:18 model demonstrates its behavior with adaptive reactions. With the two vibration plates, the model is set into oscillations, which are actively compensated by the actuated columns and diagonals. It is also used to record the movements by camera and to develop a redundant surveillance system. The adaptive behavior of the 1:18 model and the demonstrator high-rise is coordinated via system control. Shown on the left, the developed system model controls the actuators based on the incoming sensor data. The system model and 1:18 model are used to analyze occurring errors and to develop a failure-safe system, as shown on the far left.

The models of the vertical and horizontal axis have fundamentally different characteristics. The architectural models refer to a whole, the vision of the realized and contextualized demonstrator high-rise. The initial architectural models can be understood as a concretion, as they reify an idea for the first time. Both, the first acrylic glass model and also the second wooden model with a tower surrounded by transparent paper define the volume spatially. In their abstract, sometimes exaggerated design, they bear manifold references not only to place, local building culture, use, but also to architectural history. Both models are set in the urban context; the augmented reality model is also best viewed at the building site. All three involve the surroundings and open up perspectives for the future high-rise and possible qualities, shape, and use. The architectural design is formative for the further design process, as the first model is a positioning and functions as a vision and role model for the further interdisciplinary design process. The following functional models reduce the architectural conception to the benefit of a function, they concretize in particular the adaptive dimensions of the architectural design. It becomes apparent that architectural models follow a holistic approach, while functional models reduce the overall design to execute and represent the adaptive behavior.

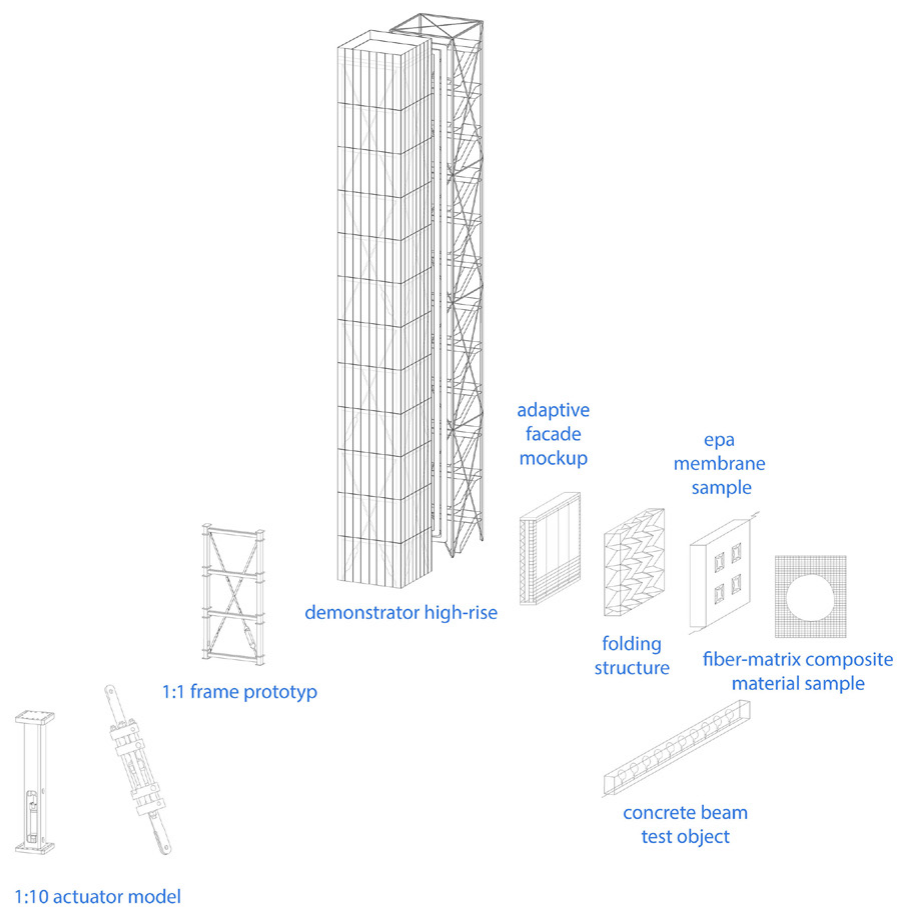

Figure 5. Model cosmos with adaptive components for the demonstrator high-rise.

\subsection{Models for the development of adaptive components}

In second consideration of the model cosmos, the models found in this study, which were created in the course of the development of various adaptive components for the demonstrator high-rise building, are discussed (Figure 5). The 1:1 frame prototype on the left has one quarter of the height and the façade width of the demonstrator building. It was built to test the functionality of the actuated components on a structural unit. As planned for the high-rise, the developed actuators are serially integrated into the diagonals and connected in parallel inside the steel column. With the construction of the frame prototype, the system of the activated structure was successfully tested and the measurement data could validate the static calculations. The researchers improved the design of the actuators for 
the high-rise on the basis of the knowledge gained from the 1:1 frame. In the development of the actuators, compressive forces from the hydraulics had to be redirected into tensile forces for the diagonals. The researchers found that the 1:10 models of the actuators from the 3D printer conveyed the functional principle much better than renderings.

The high-rise design allows adaptive components to be gradually installed and tested such as adaptive façades, as shown on the right. A first façade project looks for smart materials and technical controls to replace the properties of the missing mass in lightweight construction. Concepts for adaptive façade elements are first evaluated in terms of building physics in a combination of different software, since these can only simulate fixed states and not their transitions. Before realization, mockup models illustrate the functional principle, e.g. a temperature-dependent surface change from light to dark. A second project develops sandwich façade elements with internal folding structures, which must first undergo endurance testing. The tested folded structures indicate possible fractures and are used to demonstrate the folding principle. A further project is experimenting with electroactive polymer actuators (EPA) for switchable breathability in building skins. The EPA membrane samples prove the possible production and control before function-optimized prototypes are fabricated. In addition, sensory and transparent fiber-matrix composite materials are developed for gas-filled and adaptive façade cushions. Based on chemical calculations, material samples are prepared, whose analysis and findings improve further samples. Gradually, the desired adaptive material properties are approached. All functionally optimized façade systems will be installed and tested on the demonstrator high-rise. This also includes concrete beams with integrated fluid actuators, whose functionality and resiliency are examined with test objects. While a simulation is mathematically exact, tolerances arise during the practical implementation, which explains why physical functional models are necessary. Deviations allow researchers to draw conclusions on inaccurate simulation, on large tolerances in the implementation or wrong assumptions.

The study shows that it is an important integrative feature of the architectural design that the high-rise is open for the successive extension of adaptivity. That means, the installation of the developed adaptive components one after the other. In the development of those components, concepts and technical calculations are transformed into models or samples. In the study it becomes clear, that while mockups only illustrate the adaptive principle, functional models and material samples confirm and prove the possible production and perform an adaptive function. The imperfect samples provide the researchers with important information for possible improvements in calculation or implementation. With each new test, the researchers approach the desired adaptive material properties and qualities. With the installation of the adaptive façades and beams in the demonstrator high-rise, a complete measurement validation becomes possible. As a result, the degree of adaptation of the adaptive high-rise building gradually increases.

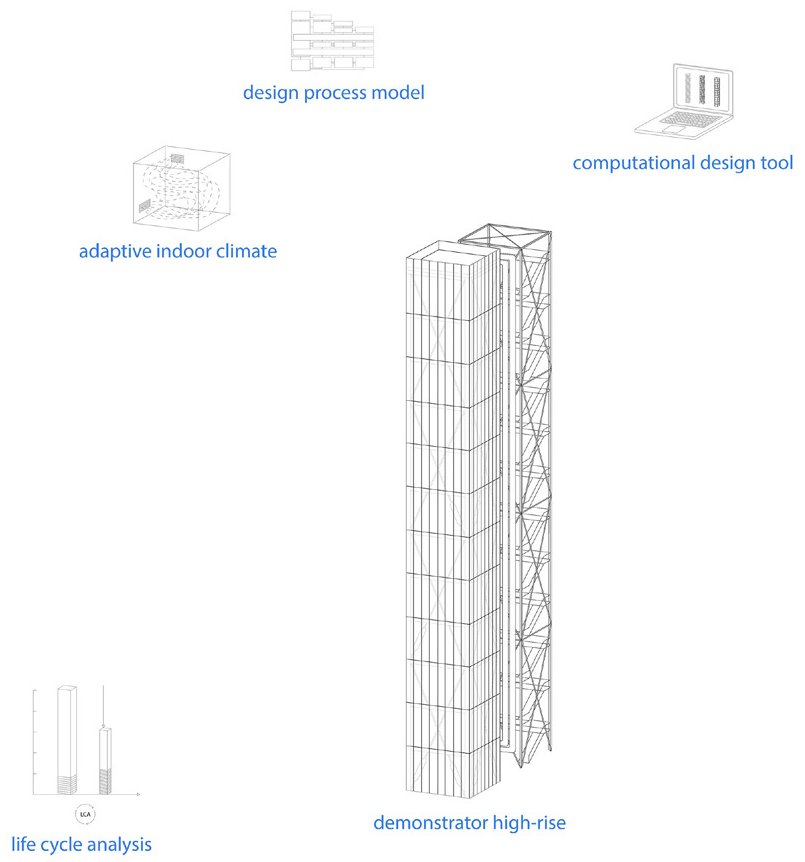

Figure 6: Model cosmos with analytic projects of the demonstrator high-rise.

4.3 Theoretical, reflective and projective projects on the demonstrator high-rise

The third category shows analytical models found in the study that reflect the design, planning and development 
process of the high-rise or treat it as a case study (Figure 6). The complex design and planning process of the demonstrator high-rise is reflected in a process model and recommends early multidisciplinary cooperation between architecture, structural design, system dynamics and mechanical engineering for adaptive building (top left). As shown on the right, a parameter-based computational design tool is developed to integrate the different and sometimes contradictory adaptation goals into the design process from the very beginning. For this purpose, optimization algorithms developed on the high-rise are integrated into the program, but also autonomous design interventions by architects are made possible. In the third project, the ecological sustainability of the high-rise is balanced within the framework of the life cycle analysis (LCA), and it is examined to see whether a driven structure is more sustainable than a conventional one, whereby adaptive operation must also be included in the calculation. For this purpose, researchers determine the $\mathrm{CO} 2$ balance of building materials, construction, operation phase and recycling in the calculation model. As a fourth project, the development and control of an adaptive indoor climate is shown, which is linked to research on adaptive façades. The adaptive indoor climate reacts to individual user needs and environmental conditions, first in the simulation and later on one floor in the demonstrator high-rise. Finally, our own subproject should be mentioned here, since the model cosmos reflects the use of different model types and their relations to each other as well as their contribution to the implementation of the high-rise.

The analytical models found in the study are not only used to reflect and visualize processes but also to calculate and simulate procedures. Thereby, the analytical models indicate that the entire research project can be understood as a model from whose developments and findings, algorithms, computer programs, technical systems, security standards, standardization, methods and process models are derived. The publications of the various developments and considerations have the effect that the adaptive high-rise is already placed in the research discourse before its implementation. The built demonstrator high-rise will in turn be a "model" for other adaptive buildings.

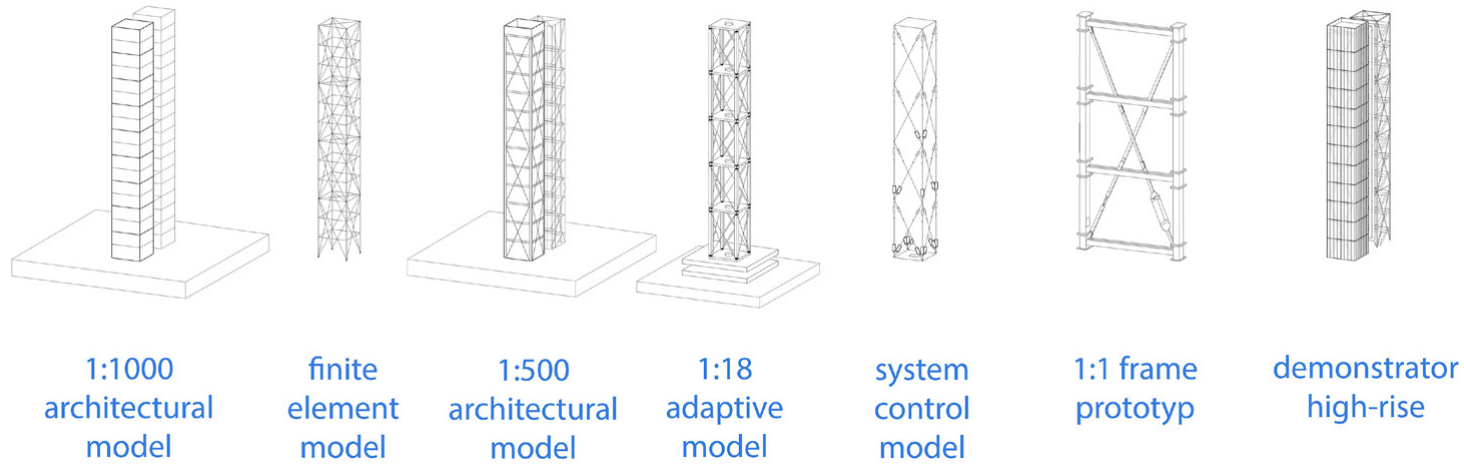

Figure 7: Design process with architectural and functional models of the demonstrator high-rise.

\section{Design and development models in the adaptive design process}

The architectural design and development process of adaptive structures runs in parallel, so architectural planning must be open to the progress of the disciplines involved. The architectural models (Figure 7, model 1 and 3) show the tower as mass and space, the second one with a statement about the construction. The structural models $(2$ and 5) describe static or control systems and are mathematically functional models. In the 1:18 model and the 1:1 frame prototype (4 and 6), the developments from building construction, mechanical engineering and control technology come together, for a statically adaptive reaction. All models gradually contribute to the implementation of the adaptive high-rise structure (7).

The first architectural model, as a role model, determines the further design process. The qualitative evaluation of the architectural models is based on architectural, spatial and aesthetic criteria. The architectural models refer to a planned whole, the demonstrator high-rise, and are open and intended to integrate the upcoming (technically) developments and detailing of other disciplines. The functional models considerably reduce the architectural design in order to develop selected functions, movements or behaviors. As activated models, they perform or simulate the behavior of the adaptive structure for calculation and dimensioning, development and control, and for investigation. Therefore, functional models are always quantitatively evaluated and optimized accordingly.

Regarding the adaptive design in the analyzed project, the architects are responsible for the holistic consideration and form finding, understood as an initial vision; the necessary integrated functional, systemic and technical solutions are developed in close interdisciplinary cooperation. In this project, the adaptive behavior is currently only carried out by the functional models and has been omitted in the architectural models. In future adaptive architecture projects this could change. Associated with this, new tasks for both the architectural models and the architecture become apparent. Adaptive architecture needs new or additional models that perform. In 
addition to the determined models, dynamic models are needed that show changing states of adaptation.

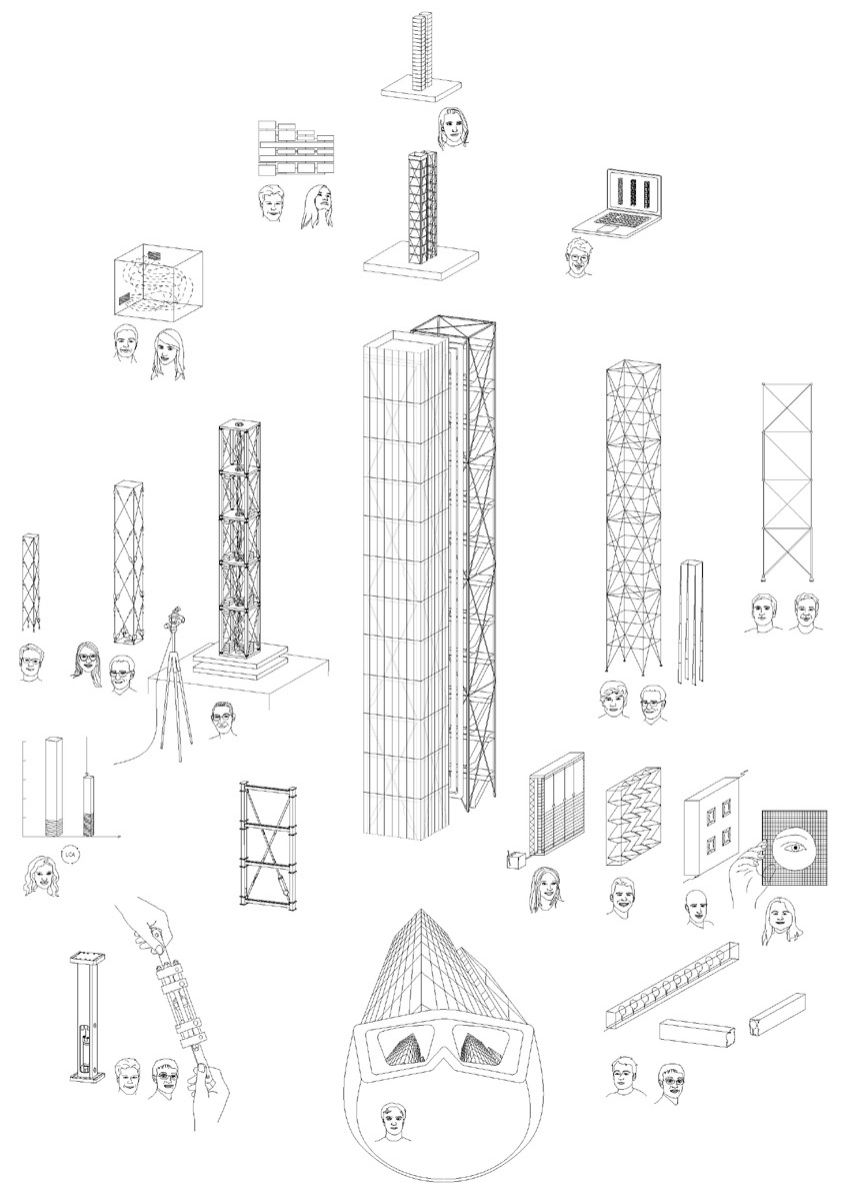

Figure 8. Models and researcher in the multidisciplinary design network.

\section{Models as significant actors in the multidisciplinary project development}

In addition to their direct contribution to the design, development and realization of the demonstrator high-rise, the models have further essential functions and tasks that are important for the success of the project. These are soft and inherent dimensions that they take on as non-human actors and which are revealed in the joint consideration with the researchers in the research process (Figure 8). The models and their images also have an essential social dimension. The holistic architectural design approach marks the beginning of the project and unites researchers from various disciplines because the overall architectural idea provides orientation and motivation for all participants by formulating a common vision. While the research process continues, all other models and their illustrations remain as important milestones in the project development.

The design process is a multidimensional, simultaneous and collaborative development with various discipline-specific contributions to the implementation of the high-rise. Therefore, the architectural design is open so that a joint development and detailing is possible. In the adaptive high-rise project, the early cooperation of the disciplines, especially architecture, structural mechanics and system dynamics, was new for everyone, and thus also the chronological coordination and specialized communication. But particularly for adaptive architecture, close and early interdisciplinary cooperation promotes well integrated design solutions (Honold, Leistner et al. 2019). All models in the model cosmos stand for the multidisciplinary tasks and cooperation to realize the adaptive high-rise. 


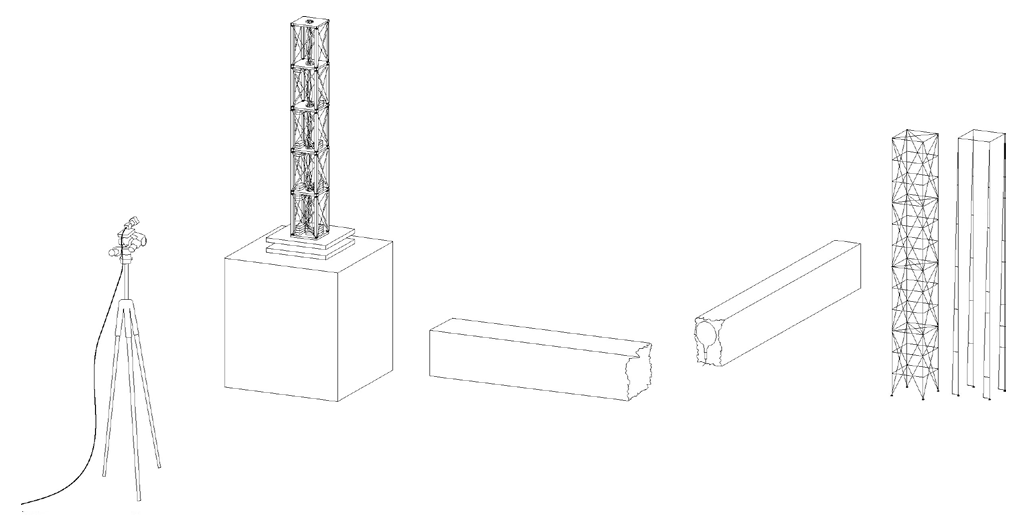

Figure 9. Filming the behavior of the 1:18 model, testing the beam with fluid actuators, interpreting the results of the structural analysis.

In the investigated high-rise project architectural and functional, simulative and analytical models are used. Each model is preceded by theoretical considerations, assumptions, calculations and speculations. While the theory can be understood as the respective discipline-specific language, such as calculations or equations from chemistry, statics, building physics, mathematics or system dynamics, the results are abstract numerical values, chemical elements, heat-transmission coefficients, algorithms or integral equations. With the simulative or physical transformation into a model or material sample the result is taking on a commonly understandable form. Theory is transferred into practice and new knowledge is gained and fed back to further the procedure and carry it across disciplines. This also applies to the digital: researchers are using animated simulation models to better understand the movements or functions of the system or are using a colored beam model to more easily interpret the structural analysis results (Figure 9). In conclusion, this means models translate abstract processes and results and are therefore a tool of knowledge emergence. In multidisciplinary exchange, the models and their illustrations serve the purpose of communication and facilitate the exchange of knowledge as they function similar to a common language. Therefore, we conclude that models can assume the role of translators in a multidisciplinary architectural design project.
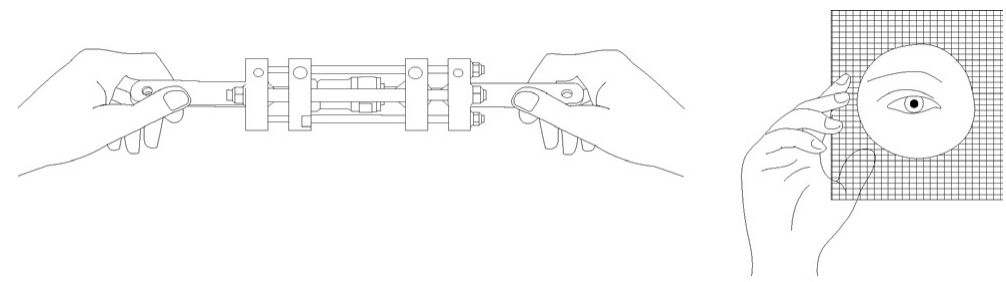

Figure 10. Moving the 1:10 model of the actuators, perceiving the degree of transparency of a material sample.

Models contribute to communication in a special way, since they make knowledge accessible in a multisensory, cross-disciplinary way. The study reveals that those who move the model with their own hands understand the functional principle of a folding structure or the actuator intuitively and better than through a visualization. In handling, the inherent knowledge of the models becomes accessible and the functional principle becomes understandable (Figure 10). Furthermore, the material sample is not only the practical implementation of the chemical equation. The elasticity is felt manually, and those who look through the sample can perceive the degree of transparency. It becomes apparent that modeled knowledge is experienced directly, and active understanding is supported. In a multidisciplinary research project, models contribute significantly to knowledge genesis and communication. Similar to participating researchers, models are actors within knowledge cultures that together form a network of insights and developments (Figure 8).

The models analyzed in this study are essential carriers of interdisciplinary cooperation. The models are just as involved in the design, research and development process of the demonstrator high-rise as the researchers themselves. In joint project development, it is not enough just to communicate finished results, it is essential to explain a context precisely. These subject-specific contributions are never successfully explained by abstract calculations, but always with the help of different models. Only by sharing the challenges and insights through models can an interdisciplinary discussion and further development of the overall project be achieved. In contrast to the previous one-sided detailing by architects and the subsequent correction by specialist planners, adaptive solutions are developed in a multidisciplinary way. 


\section{Results and conclusion}

The model study from the architectural design to the realization of an adaptive high-rise building in a multidisciplinary research project shows, as a result, that models of different kinds are significantly involved in the design and development, in knowledge creation and transfer as well as in communication. The investigations highlight that models are the essential medium for multidisciplinary cooperation because they speak an interdisciplinary language and function as translators between different knowledge cultures. In multidisciplinary cooperation, models serve less for external presentation; rather, as problem-describing models or imperfect material samples, they promote the joint solution of technical and design challenges. Architectural models also take on new tasks and reveal further qualities. In the multidisciplinary project, the initial architectural design idea serves as a role model and vision for subsequent development processes, both in terms of the orientation of the design project and the motivation of the team. The architectural model is a concretion that functions as a positioning for the further project. The subsequent functional models reduce the architectural design idea in order to develop and demonstrate adaptive functions, while analytical models reflect and evaluate the design process or project further implementations. The case study was carried out in a Collaborative Research Center focusing on future construction; as long as adaptive building technology is still evolving, many of the model types found in this study are necessary in architectural practice.

The investigation shows that, for adaptive buildings, new roles and tasks of models emerge in the architectural context exceeding and significantly expanding our idea and understanding of classical architectural models. We saw that adaptive architecture requires additional and new models to perform and act. Parallel to or instead of conventional form-finding models, dynamic models are needed that show changing states of adaptation. The model study already shows possibilities and challenges of adaptive technology for architectural production, whose set conditions are changing significantly. A new design approach in architecture is evolving and will require the use of models that perform adaptive states. In order to gain further insights into this adaptive design approach, we will investigate other architectural projects combined with our own speculative design scenarios to gain additional information about future model types and roles in the design, planning, implementation and use of adaptive buildings. We will thereby focus specifically on the different forms of interaction with and operation of models through architects and engineers.

The study takes a look ahead at the innovations of the future. Adaptive architecture, as developed in the research project with the demonstrator high-rise, not only fundamentally changes our idea and experience of buildings, but also the work of architects and engineers. In the recent past, computer technology has significantly changed design, planning and manufacturing. These are methodological changes that lead to more complex architectural forms, but the buildings remain relatively fixed and determined. The adaptive, especially concerning load-bearing structures, as in the analyzed project, announces a fundamental change in building culture, comparable to Modernism, when the separation of static structure and spatial design gave architects new design opportunities. Based on the model study we see a fundamentally new approach in the design attitude which does not aim at an optimum for a certain situation, but considers several situations. The architects of the future will create active architectural environments that dynamically adapt to space, location, use and form.

\section{References}

CRC, Collaborative Research Centre 1244 (2017). Adaptive Skins and Structures for the built Environment of Tomorrow. [Online] Available: https://www.sfb1244.uni-stuttgart.de/en/index.html (January 26, 2020)

Döring, N., Bortz, J. (2016). Forschungsmethoden und Evaluation in den Sozial- und Humanwissenschaften. Berlin, Heidelberg: Springer.

Fox, M. (2016). Interactive architecture: Adaptive world. New York: Princeton Architectural Press.

Helfferich, C. (2014). Leitfaden und Experteninterviews. Handbuch Methoden der empirischen Sozialforschung, ed. N. Baur, J. Blasius, Wiesbaden: Springer Fachmedien, DOI 10.1007/978-3-531-18939-0_39.

Hill, J. (2018). Skyscraper: Vom Tribune Tower in Chicago bis zum Burj Khalifa in Dubai. München: Deutsche Verlags-Anstalt.

Honold, C., Leistner, S., et al. (2019). Requirements in the design phase of the integral planning process of adaptive buildings. SSP

ICD, Institute of Computational Design (2013). HygroSkin: Meteorosensitive Pavilion. University of Stuttgart, [Online] Available: https://www.icd.uni-stuttgart.de/projects/hygroskin-meteorosensitive-pavilion/ (January 26, 2020)

Kolarevic, B., Parlac, V. (2015). Building dynamics: Exploring architecture of change. Abington, Oxon, New York: Routlegde, p.7.

Schilling, A. (2018). Architecture and model building: concepts - methods - materials. Basel, Birkhäuser.

Schmidt III, R., Austin, S. (2016). Adaptable architecture: Theory and practice. London; New York: Routledge. Sobek, W. (2016). Ultra-Lightweight Construction. GAM 12: Structural Affairs: Opportunities and Perspectives for Cooperation in Planning, Design and Construction, edited by A. Fuchs. (Basel: Birkhäuser, 156-167. 
Ulber, M. (2017). Landschaft und Atmosphäre. Künstlerische Übersetzungen. Bielefeld: Transcript.

Ulber, M., Mahall, M. (2019). Adaptive Architecture as Mediator between Humans and Earth. AGATHÓN International Journal of Architecture, Art and Design, n. 06, pp. 94-103, DOI: 10.19229/2464-9309/692019

Ulber, M., Mahall, M., Serbest, A. (2020). (In)stable Boundaries - towards Adaptive Architecture: Interrelated changes in Architecture, Atmosphere and human Experience. Environment, Space, Place, University of Minnesota Press journal.

Ursprung, P. (2012). Exposed Experiments: Herzog \& De Meuron's Models. The Architectural Model - Tool, Fetish, Small Utopia, ed. O. Elser, P. Cachola, T. Schmal, T. Fankhänel, P. Bausch, L. Rosenblatt, Zürich: Scheidegger \& Spiess, 51-56.

Yaneva, A. (2005). Scaling Up and Down: Extraction Trials in Architectural Design. Social Studies of Science 35, no. 6, London: Social Studies of Science - SOC STUD SCI, 867-94, doi: 10.1177/0306312705053053.

Yaneva, A. (2009). The Making of a Building. A Pragmatist Approach to Architecture. Oxford: Lang.

Yiannoudes, S. (2016). Architecture and adaptation: From cybernetics to tangible computing. New York: Routledge.

Zumthor, P. (2006). Atmospheres: Architectural environments - surrounding objects. Basel: Birkhäuser.

\section{List of Images}

Figure 1. (C) Institute for Lightweight Structures and Conceptual Design (ILEK), University of Stuttgart. Figure 2-10. (C) Marie Ulber.

Marie Ulber, PhD is a Postdoctoral Associate in the Collaborative Research Centre Adaptive Skins and Structures for the Built Environment of Tomorrow at the HafenCity University Hamburg, Germany. She studied architecture, received her doctorate in Art and Design on landscape and atmosphere, and analysed architecture and atmosphere in a postdoctoral project. Her research focuses on various relationships and correlations between humans and the (built) environment; published in a book "Landschaft und Atmosphäre - Künstlerische Übersetzungen". E-mail: marie.ulber@hcu-hamburg.de

Mona Mahall is a Professor of Architecture and Art at the HafenCity University Hamburg (Germany). She works as a collective at the intersection between art and spatial practices. Through a feminist lens they reflect and negotiate the evolving cultural, social, political, and technological relations and processes that constitute space, be that analogue or digital. Their research-based projects include various media such as models, installations, scenographies, (video-) texts, concepts, and publications. Her projects and research are exhibited and published internationally, among others the monograph "How Architecture Learned to Speculate" (with Asli Serbest). She is a Member on the Board of the CRC 1244 Adaptive Skins and Structures. E-mail: mona.mahall@hcu-hamburg.de

Asli Serbest is a Professor of Temporary Spaces at the University of the Arts Bremen (Germany). Together with Mona Mahall, she works as a collective at the intersection between art and spatial practices. Her focus is on particular past, present and future contexts to negotiate the evolving relationship between the arts, space, media, and curatorial practices. Her projects are exhibited internationally, among others the Biennale di Venezia, Pinakothek der Moderne Munich, Storefront for Art and Architecture New York. In 2019, she co-curated (with Mona Mahall) the 7th International Sinop Biennial under the title of "A Politics of Location." E-mail: asli.serbest@hfk-bremen.de

\section{Acknowledgements}

The authors gratefully acknowledge the generous funding of this work by the German Research Foundation (DFG - Deutsche Forschungsgemeinschaft) as part of the Collaborative Research Centre 1244 (SFB) „Adaptive Skins and Structures for the Built Environment of Tomorrow“/project A03 - Architectural design Concepts for adaptive Skins and Structures. Furthermore, the authors would like to thank all researchers of the Collaborative Research Center 1244 of the University of Stuttgart for their support, interviews and contributions to this study. They also like to thank Sarah Wilhelm and Johanna Dorn for the drawings of the model cosmos on the computer. 